Document downloaded from:

http://hdl.handle.net/10251/161857

This paper must be cited as:

De Luis-Fernández, B.; García-Fernández, A.; Llopis-Lorente, A.; Villalonga, R.; Sancenón Galarza, F.; Martínez-Máñez, R. (2020). A 1-to-2 demultiplexer hybrid nanocarrier for cargo delivery and activation. Chemical Communications. 56(69):9974-9977.

https://doi.org/10.1039/d0cc03803b

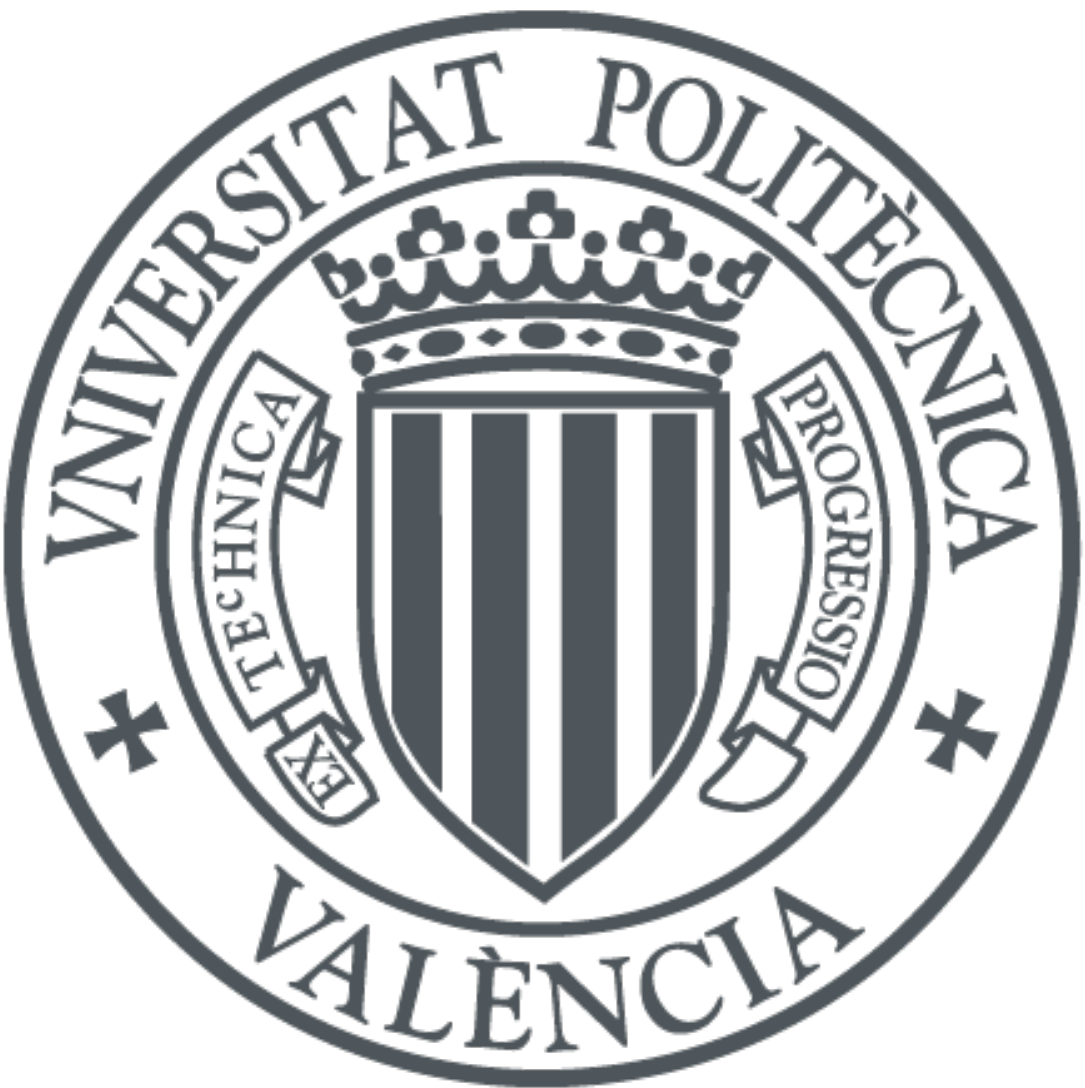

The final publication is available at

https://doi.org/10.1039/d0cc03803b

Copyright The Royal Society of Chemistry

Additional Information 


\title{
A 1-to-2 Demultiplexer Hybrid Nanocarrier for Cargo Delivery and Activation
}

\author{
Beatriz de Luis, ${ }^{a, b}$ Alba García-Fernández, ${ }^{a, b, c}$ Antoni Llopis-Lorente, ${ }^{a, b}$ Reynaldo Villalonga, ${ }^{e}$ Félix \\ Sancenón, ${ }^{a, b}$ and Ramón Martínez-Máñez ${ }^{* a, b, c, d}$
}

\begin{abstract}
A biocomputing strategy implemented in hybrid nanocarriers for controlled cargo delivery is described. The nanodevice consists of enzyme-functionalized Janus Au-mesoporous silica nanoparticles which behaves as an electronic demultiplexer (DEMUX). The nanocarrier is capable of reading molecular information from the environment (lactose) and selecting one of two possible outputs (galactose production or 4-methylumbellilferone release and activation) depending on the presence of an addressing input (NAD ${ }^{+}$.
\end{abstract}

The research in the field of nanobotics is a current topic in science since they offer promising tools in a great number of different areas including diagnostics and therapeutic strategies. ${ }^{1}$ Futuristic nanobots are expected to have specific functionalities such as (i) being able to read information from the environment, (ii) having autonomous movement and (iii) being able to communicate with other nanobots for instance through the interchange of chemical messengers. ${ }^{2}$ In this scenario, an attractive characteristic in nanoparticles is to design them so that they are able to sense the environment and act accordingly. Although simple controlled release nanoparticles already perform this action, since they deliver a cargo on-command in the presence of certain stimuli, it is desirable in this field to design more advanced nanosystems capable of performing complex biocomputing operations.

Biocomputing science, which studies biocompatible reactions utilized for information processing and signal

\footnotetext{
a. Instituto Interuniversitario de Investigación de Reconocimiento Molecular y Desarrollo Tecnológico (IDM), Universitat Politècnica de València, Universitat de València, Camino de Vera s/n, 46022 Valencia, Spain. E-mail: rmaez@qim.upv.es b. CIBER de Bioingeniería, Biomateriales y Nanomedicina (CIBER-BBN), Spain.

c. Unidad Mixta UPV-CIPF de Investigación en Mecanismos de Enfermedades y Nanomedicina, Universitat Politècnica de València, Centro de Investigación Príncipe Felipe, Valencia, Spain.

d. Unidad Mixta de Investigación en Nanomedicina y Sensores, Universitat

Politècnica de València, Instituto de Investigación Sanitaria La Fe, Valencia, Spain.

e. Nanosensors \& Nanomachines Group, Department of Analytical Chemistry,

Faculty of Chemistry, Complutense University of Madrid, Madrid, Spain.
}

transduction into YES/NO decisions, is gaining increasing attention. ${ }^{3}$ This behavior mimics the binary $(0,1)$ format of Boolean logic operations in electronics. Up to now, different discrete and sequential logic gates based on enzymatic reactions, fluorescence or absorption switches and DNA crosslinking, cleavage or strand displacements among others have been developed.4-7 These are capable of logically processing multiple input signals into a final YES/NO output. However, few of such systems have been implemented in nanodevices for controlled-release applications. Moreover, considering the environmental complexity at the cellular level it is reasonable to consider that biocomputing networks of nanodevices with builtin logics are required to successfully process all the available information and achieve a desired final action.

With the aim of broadening the scope and exploring the implementation of biocomputing operations in stimuliresponsive delivery applications, herein we report the design and preparation of a nanodevice that imitates a 1-to-2 demultiplexer reading the molecular information from the environment and selecting a response. Demultiplexers (DEMUX) are digital electronic components used to switch signals between different channels in a circuitry. ${ }^{8-11}$ 1-to-2 demultiplexers process 1 input and select only one of the possible 2 outputs depending on the presence or the absence of an additional input (addressing input). It can be implemented by connecting two AND logic gates operating in parallel and one NOT logic gate (Scheme 1A). Previous work from Willner and coworkers showcased the realization of a DNA-based demultiplexer for the assembly of DNAzyme nanostructures. ${ }^{11}$ Here, we have implemented this behaviour by the programmed concatenation of enzyme-based reactions implemented in a drug delivery nanocarrier conferring the ability to analyze potential physiological scenarios and actuate (cargo release) only when needed. Besides, taking advance of the efficiency and versatility of the enzymes, the whole mechanism is complemented with the extra functionality of in situ cargo activation once delivered. ${ }^{12}$ In this way, we expand the utility of mesoporous carriers by introducing different biocomputing 


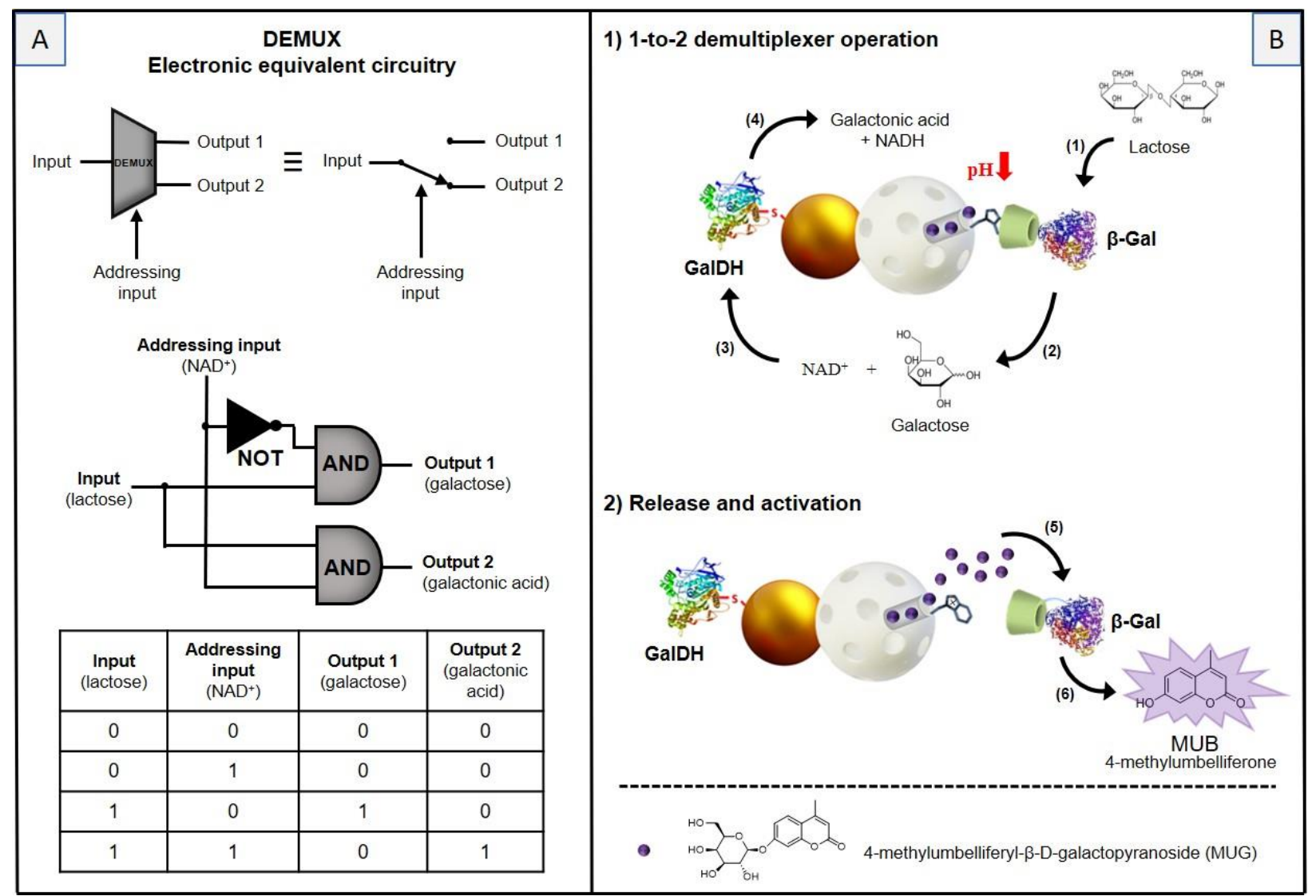

Scheme 1. (A) In descending order: representation of a 1-to-2 demultiplexer following electronic symbology and the equivalent switching device, representation of the equivalent combinatorial logic circuitry and the corresponding Truth Table that describes the behaviour of the system. (B) Illustration of the nanodevice concatenated three-step enzymatic cascade when the input (lactose) and the addressing input (NAD+) are present leading to MUG release and subsequent activation into the strong fluorescent MUB.

elements which can be integrated in a complex nanodevice, as a versatile strategy for the design of nanosystems with logic programmable behaviour. Whereas typical nanocarriers release their cargo in the presence of a single stimulus, nanocarriers with biocomputing demultiplexer capabilities could be useful for the design of more selective delivery systems targeting certain cells or tissues where two stimuli are simultaneously present. For instance, cancer cells have been demonstrated to exhibit elevated levels of both saccharide uptake and $\mathrm{NAD}^{+} / \mathrm{NADH} \cdot{ }^{13,14}$

The nanocarrier $\left(\mathrm{S} 1_{\mathrm{enz}}\right)$ is based on Janus Au-mesoporous silica nanoparticles which are functionalized with the enzyme galactose dehydrogenase (GalDH) on the gold face. The mesoporous unit is loaded with a model pro-fluorophore (4methylumbelliferyl- $\beta$-D-galactopyranoside, MUG) and capped with a $\mathrm{pH}$-responsive supramolecular nanovalve consisting of an inclusion complex between a benzimidazole derivative and $\beta$-cyclodextrin (see Scheme S1, ESI). The enzyme $\beta$ galactosidase ( $\beta$-Gal) attached to the $\beta$-cyclodextrin completes the design.

The operation of the final $\mathrm{S} 1_{\mathrm{enz}}$ nanodevice is depicted in Scheme $1 \mathrm{~B} . \mathrm{S} 1_{\mathrm{enz}}$ is able to sense the presence of lactose (1) which is transformed into glucose and galactose by the enzyme $\beta$-Gal (2). The generated galactose (3) is the "output 1 ". Moreover, when the enzymatic cofactor $\mathrm{NAD}^{+}$is also present in the environment, the galactose is oxidized to galactono- $\gamma$ lactone by the GalDH enzyme on the Au face. This lactone is spontaneously converted to galactonic acid (4) which is the "output 2". When only "output 1 " is generated no cargo delivery is observed, however, if "output 2" is obtained (galactonic acid) a local drop of the $\mathrm{pH}$ causes the protonation of benzimidazole groups $\left(\mathrm{pK}_{\mathrm{a}}=5.55\right)^{15}$ and the dethreading of the inclusion complex triggering the delivery of the entrapped MUG (5) from the mesoporous face. In addition, an additional cargo activation is accomplished by the hydrolysis of the glycosidic bond of the released MUG by the $\beta$-Gal enzyme inducing the appearance of a high fluorescent emission (6) due to the formation of 4-methylumbellilferone (MUB). This activation step might be crucial in situations where the payload needs to be derivatized to overcome solubility and stability troubles during the device assembly but reactivated when delivered in the target site. ${ }^{16}$

The Janus Au-mesoporous silica nanoarchitecture allowed to anchor two different enzymes to the same nanodevice (one on the $\mathrm{Au}$ face, other on the silica face) using different chemistries. In our system, the importance of the selected strategy relies on two main factors: (i) there is more space for $\beta$-Gal immobilization (which is less active than GalDH) on the benzimidazole-mesoporous surface; and (ii) GalDH, which is commercialized in much lower quantities (as compared to $\beta$ Gal), can be directly immobilized on the Au without the need of previous derivatization. Based on enzymatic assays (see ESI), the amounts of $\beta$-Gal and GalDH on $S 1_{\text {enz }}$ were determined to be 51 and $13 \mathrm{mg}$ per $\mathrm{g}$ of nanoparticle, respectively (i.e., 7:3 
$\beta$ Gal:GalDH ratio); with lower $\beta$-Gal activity $\left(0.007 \mathrm{U} \cdot \mathrm{mg}^{-1}\right)$ as compared to GalDH (1.05 U. $\left.\mathrm{mg}^{-1}\right)$.
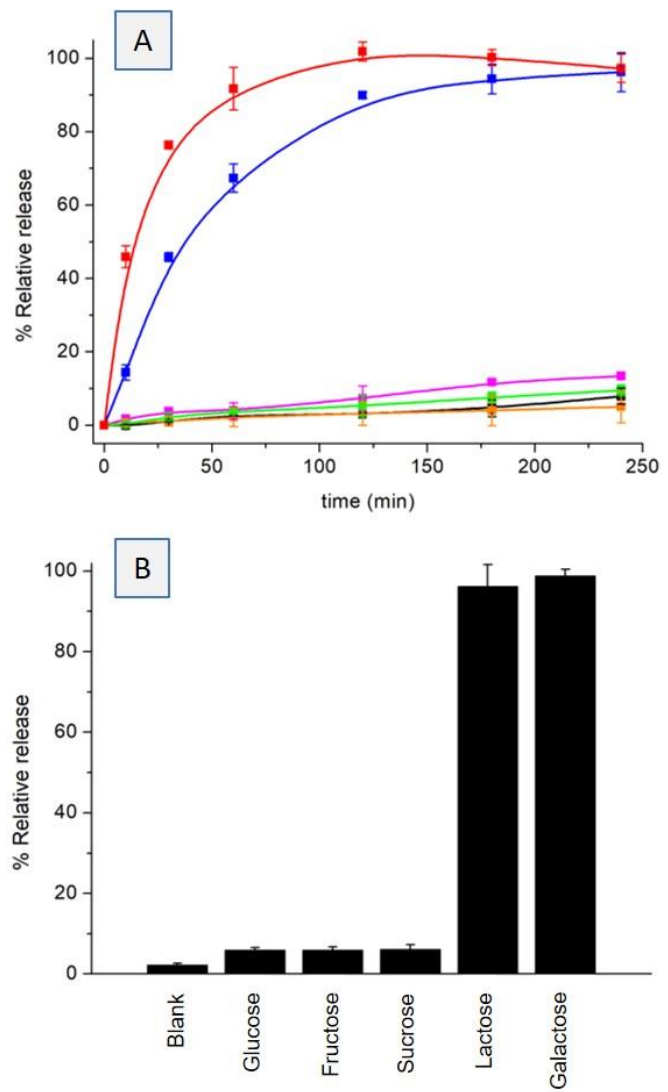

Figure 1. (A) Normalized controlled release kinetic profiles obtained for solid $\mathrm{S} 1_{\mathrm{en}}$ in the absence of stimulus (blank, black curve); in the presence of $\mathrm{NAD}^{+} 1 \mathrm{mM}$ (pink), in the presence of galactose $1 \mathrm{mM}$ (orange); in the presence of lactose 1 $\mathrm{mM}$ (green) and in the presence of both lactose and $\mathrm{NAD}^{+} 1 \mathrm{mM}$ (blue) and galactose and $\mathrm{NAD}^{+} 1 \mathrm{mM}$ (red). (B) Normalized cargo release from $\mathrm{S} 1_{\text {enz }}$ in the presence of $\mathrm{NAD}^{+} 1 \mathrm{mM}$ and different saccharides at $1 \mathrm{mM}$ after $4 \mathrm{~h}$.

After the synthesis and characterization of the nanodevice $\mathrm{S} 1_{\text {enz }}$ using standard techniques (see ESI) the 1-to-2 demultiplexer behaviour was examined. For this purpose, $\mathrm{S} 1_{\mathrm{enz}}$ $\left(1 \mathrm{mg} \cdot \mathrm{mL}^{-1}\right)$ was suspended in an aqueous solution at $\mathrm{pH} 7.5(20$ $\mathrm{mM}$ sodium sulphate) in the absence and in the presence of selected stimuli (lactose, galactose, $\mathrm{NAD}^{+}$, galactose $+\mathrm{NAD}^{+}$, lactose $+\mathrm{NAD}^{+}$) and the emission of the generated MUB at 445 $\mathrm{nm}\left(\lambda_{\mathrm{ex}}=365 \mathrm{~nm}\right)$ was measured. In the absence of stimuli (black curve in Figure 1A) a low MUB emission was observed (less than $10 \%$ of the maximum observed) which is ascribed to some remaining not fully capped pores. A negligible MUB fluorescence was also observed when $\mathrm{S} 1_{\mathrm{enz}}$ was suspended in the presence of only galactose (orange curve), only lactose (green curve) and only $\mathrm{NAD}^{+}$(pink curve), all of them at $1 \mathrm{mM}$ concentration. As a clear contrast, a marked MUG release/activation from $\mathrm{S}_{1}$ enz was observed in the presence of the addressing input $\mathrm{NAD}^{+}$and lactose (both at $1 \mathrm{mM}$ ) (Figure $1 A)$, which is ascribed to the triggering of the whole programmed enzymatic pathway as shown in Scheme 2B. Moreover, we also observed that when galactose (instead of lactose) and $\mathrm{NAD}^{+}(1 \mathrm{mM})$ were used as inputs, MUG release and activation are achieved faster (ca. 90\% after $60 \mathrm{~min}$ ) (red curve in Figure 1A). This faster response is in agreement with the omission of the first step of the enzymatic cascade. In this case dethreading of the supramolecular complex and pore opening is produced via direct transformation of galactose into galactonic acid by GalDH without depending on the previous activity of $\beta$-Gal. In additional control experiments, $\mathrm{S} 1_{\mathrm{enz}}$ response was tested in the presence of other saccharides (glucose, fructose and sucrose at $1 \mathrm{mM}$ ) and setting the addressing input concentration $\left(\mathrm{NAD}^{+}\right.$) to $1 \mathrm{mM}$. In all cases negligible cargo delivery after $4 \mathrm{~h}$ was observed (Figure 1B), pointing out the high specificity of the delivery system.

The crucial role played by both immobilized enzymes in the delivery/activation mechanism was tested using two new nanodevices lacking the enzyme GalDH $\left(S 1_{\beta G a l}\right)$ or the enzyme $\beta$-Gal $\left(\mathrm{S} 1_{\mathrm{GalDH}}\right)$. The MUG delivery/activation process was studied with $S 1_{\beta G a l}$ and $S 1_{\text {GalDH }}$ in the presence of both lactose + $\mathrm{NAD}^{+}$at $1 \mathrm{mM}$ concentration after $4 \mathrm{~h}$. It was found that only when both enzymes are immobilized on the nanodevice ( $\left.\mathrm{S} 1_{\mathrm{enz}}\right)$ the fluorophore MUB is formed, whereas in the case of $S 1_{\beta G a l}$ and $\mathrm{S} 1_{\mathrm{GaIDH}}$ nanoparticles negligible MUG release/activation was observed since the cascade enzymatic reactions is not complete (Figure 2). Additionally, in order to demonstrate the $\beta$-galactosidase-mediated activation of MUG into MUB, we studied the response of the nanodevice lacking $\beta$-galactosidase ( $\mathrm{S} 1_{\mathrm{GalDH}}$ ) by protonation of the benzimidazole subunits dropping the $\mathrm{pH}$ of the aqueous solution to 4.5. In these conditions, negligible MUB fluorescence was observed (Figure S11, ESI) since the supramolecular complex between benzimidazole and $\beta$-cyclodextrin was dethreaded but the released MUG was not activated into the highly emissive MUB derivative due to the lack of $\beta$-galactosidase. However, when $\beta$-Gal enzyme was added to the suspension, a marked MUB emission was observed. Thus, MUG activation is ascribed to the presence of $\beta$-Gal enzyme and not to changes in environmental chemical external conditions such as $\mathrm{pH}$ variations.

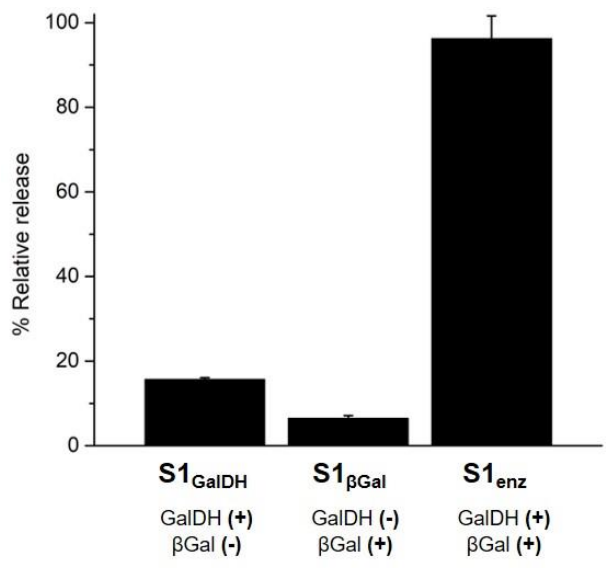

Figure 2. Normalized cargo release of different solids with $(+)$ or without $(-)$ immobilized enzymes in presence of lactose and $N A D^{+} 1 \mathrm{mM}$ after $4 \mathrm{~h}$ of addition.

Finally, we demonstrated the possible use of this 1-to-2 demultiplexer at cellular level. First, we assessed the biocompatibility of $\mathrm{S} 1_{\text {enz }}$ by cell viability assays which revealed that the nanodevice was well-tolerated by HeLa cells at concentrations up to $200 \mu \mathrm{g} \cdot \mathrm{mL}^{-1}$ after $24 \mathrm{~h}$ of exposure (Figure $\mathrm{S} 12, \mathrm{ESI})$. In a further experiment, $\mathrm{S} 1_{\mathrm{enz}}\left(75 \mu \mathrm{g} \cdot \mathrm{mL}^{-1}\right)$ was 
internalized in HeLa cells and then cells were incubated in the absence or the presence of lactose. After 24 hours, MUB associated fluorescence was assessed by confocal microscopy (Figure 3). Control untreated HeLa cells (Figure 3A) showed negligible emission upon excitation at $405 \mathrm{~nm}$ while HeLa cells incubated with $\mathrm{S} 1_{\mathrm{enz}}$ (Figure $3 \mathrm{~B}$ ) showed a residual background signal attributed to cargo delivery from some remaining uncapped pores. In contrast, a marked emission was observed when HeLa cells were treated with the nanodevice and lactose (Figure 3C, see ESI for details). In these conditions $\mathrm{S} 1_{\mathrm{enz}}$ is able to transform lactose into galactose and glucose. The galactose formed in presence of $\mathrm{NAD}^{+}$(which is a biomolecule naturally available in cells) ${ }^{13}$ started the enzymatic cascade reactions which released MUG and ultimately activated this molecule into the highly emissive MUB fluorophore as shown in Scheme 2B. Moreover, the fluorescence intensity of the confocal images of each treatment was quantified (Figure 3, below) and a 3-fold enhancement was observed for HeLa cells treated with $\mathrm{S}_{\mathrm{enz}}+$ lactose when compared to the cells incubated with the nanodevice alone $\mathrm{S} 1_{\mathrm{enz}}$.

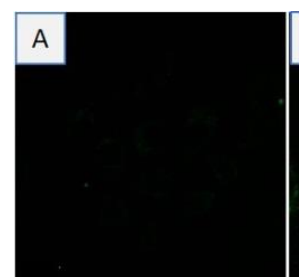

Contro

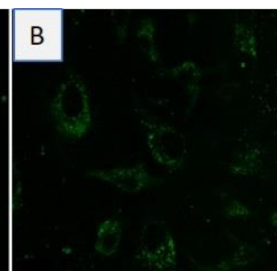

$\mathrm{S} 1_{\text {enz }}$

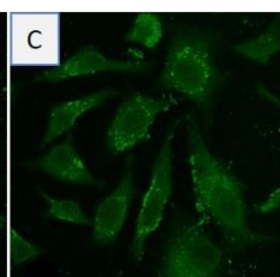

$\mathrm{S} 1_{\mathrm{enz}}+$ lactose

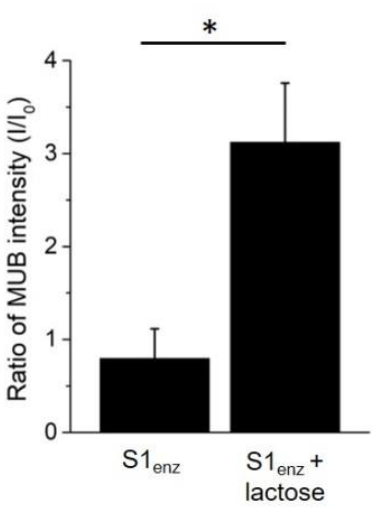

Figure 3. Top: controlled cargo release studies of $S 1_{\text {enz }}$ in HeLa cells. (A) HeLa cells without any treatment (B) HeLa cells treated with $75 \mu \mathrm{g} \cdot \mathrm{mL}^{-1}$ of $\mathrm{S} 1_{\mathrm{enz}}$ and (C) HeLa cells treated with $75 \mu \mathrm{g} \cdot \mathrm{mL}^{-1}$ of $\mathrm{S} 1_{\text {enz }}$ in the presence of lactose $1 \mathrm{mM}$. Below: ratio of mean fluorescence intensity $\left(1 / I_{0}\right)$ of MUB signal in HeLa cells for both treatments ( $\mathrm{S} 1_{\mathrm{enz}}$ and $\mathrm{S} 1_{\mathrm{enz}}+$ lactose) $(* \mathrm{p}<0.05)$.

In conclusion, we report herein the design, synthesis, characterization and operation of a new nanodevice ( $\left(\mathrm{S}_{\mathrm{enz}}\right)$ capable of reading information from the environment and processing this information imitating a 1-to-2 demultiplexer. Cargo delivery and activation is only observed in the presence of both lactose and $\mathrm{NAD}^{+}$, in a process mediated by GalDH and $\beta$-Gal. The 1-to-2 demultiplexer nanodevice is operative in aqueous solution and also when internalized in cells. Although we are aware that the illustrated system is a proof of concept, it describes a paradigm beyond the simple on-command release and activation of a cargo by leveraging organism intrinsic biomolecules. We present the use of enzyme-functionalized hybrid mesoporous nanodevices as a valuable and versatile strategy to tailor different biocomputing elements which may be implemented in individual smart nanobots acting according to environment information. ${ }^{17}$ We also envisage the interconnection of these nanodevices by means of chemical communication ${ }^{18}$ creating complex networks that operate as advanced electronic circuitries performing sophisticated executions. In the drug delivery filed, this strategy may open unexploited prospects in smart therapies and personalized treatments. ${ }^{19}$

The authors wish to thank the Spanish Government (projects RTI2018-100910-B-C41 (MCUI/AEI/FEDER,UE), CTQ2017-87954-P), the Generalitat Valenciana (PROMETEO 2018/024), the Comunidad de Madrid (IND2017/BMD-7642) and CIBER-BBN (NANOCOMMUNITY project) for support.

\section{Conflicts of interest}

There are no conflicts to declare.

\section{References}

1 F. Soto and R. Chrostowski, Front. Bioeng. Biotechnol. 2018, 6, 170.

2 X. Zhang, L. Chen, K. H. Lim, S. Gonuguntla, K. W. Lim, D. Pranantyo, W. P. Yong, W. J. T. Yam, Z. Low, W. J. Teo, H. P. Nien, Q. W. Loh and S. Soh, Adv. Mater. 2019, 31, 1804540.

3 S. Mailloux and E. Katz, Biocatalysis 2014, 1, 13.

4 E. Katz, ChemPhysChem 2019, 20, 9.

5 S. Erbas-Cakmak, S. Kolemen, A. C. Sedgwick, T. Gunnlaugsson, T. D. James, J. Yoon and E. U. Akkaya, Chem. Soc. Rev. 2018, 47, 2228.

6 H. Su, J. Xu, Q. Wang, F. Wang and X. Zhou, Nat. Commun. 2019, 10, 5390 .

7 R. Orbach, B. Willner and I. Willner, Chem. Commun. 2015, 51, 4144.

8 M. A. Arugula, V. Bocharova, J. Halamek, M. Pita and E. Katz, J. Phys. Chem. B 2010, 114, 5222.

9 J. Andreasson, S. D. Straight, S. Bandyopadhyay, R. H. Mitchell, T. A. Moore, A. L. Moore and D. Gust, J. Phys. Chem. C 2007, $111,14274$.

10 I. S. Turan, G. Gunaydin, S. Ayan and E. U. Akkaya, Nat. Comm. 2018, 9, 805.

11 R. Orbach, F. Remacle, R. D. Levine and I. Willner, Chem. Sci. 2014, 5, 1074.

12 C. Luo, J. Sun, B. Sun and Z. He, Trends Pharmacol. Sci. 2014, 35, 556 .

13 J. V. Moreira, M. Hamraz, M. Abolhassani, E. Bigan, S. Pérès, L. Pauvelé, M. L. Nogueira, J. -M. Steyaert and L. Schwartz, Metabolites 2016, 6, 33.

14 K. Adekola, S. T. Rosen and M. Shanmugam, Curr. Opin. Oncol. 2012, 24, 650.

15 G. Jerez, G. Kaufman, M. Prystai, S. Schenkeveld and K. K. Donkor, J. Sep. Sci. 2009, 32, 1087.

16 Z. Guon, Acta Pharm. Sin. B 2017, 7, 119.

17 A. Llopis-Lorente, B. de Luis, A. García-Fernández, M. Orzáez, F. Sancenón, R. Villalonga and R. Martínez-Máñez, ACS Appl. Mater. Interfaces 2018, 10, 26494

18 A. Llopis-Lorente, P. Díez, A. Sánchez, M. D. Marcos, F. Sancenón, P. Martínez-Ruiz, R. Villalonga and R. MartínezMáñez, Nat. Commun. 2017, 8, 15511.

19 A. A. Tregubov, P. I. Nikitin and M. P. Nikitin, Chem. Rev. 2018, 118, 10294. 